\title{
Adesão ao tratamento fonoaudiológico segundo a visão de ortodontistas e odontopediatras
}

\author{
Adhesion to speech therapy according to the view of orthodontists and \\ pediatric dentists
}

\author{
Cibele Pires de Moraes Varandas 1 , Leniana Guerra Campos², Andréa Rodrigues Motta ${ }^{3}$
}

\begin{abstract}
RESUMO
Objetivo: Verificar a percepção de ortodontistas e odontopediatras de Belo Horizonte e Itabira sobre a adesão à fonoterapia. Métodos: Estudo transversal realizado, por meio de aplicação de questionário, com 30 ortodontistas e 30 odontopediatras, metade de cada cidade. Na análise estatística empregou-se o Teste Qui-quadrado. Resultados: Todos dos profissionais consideram importante a atuação fonoaudiológica e encaminham com freqüência seus pacientes para tratamento, sendo que 58,3\% relataram que os pacientes necessitam de fonoterapia algumas vezes; $46,7 \%$, que os pacientes procuram o fonoaudiólogo também algumas vezes e 70,0\%, informaram que possuem um fonoaudiólogo de confiança. Verificou-se que 85,0\% informaram não haver fonoaudiólogos em seu local de trabalho e 85,0\% acreditam que trabalhar no mesmo espaço aumentaria a adesão ao tratamento. Para 75,0\%, o encaminhamento é realizado após a avaliação e 83,3\% informaram que o paciente não procura atendimento alegando falta de tempo; entretanto, 86,7\% afirmaram que pacientes com condições financeiras favoráveis costumam aderir à fonoterapia. Os encaminhamentos são realizados geralmente pelo mesmo motivo, exceto nos casos de más oclusões, não tendo sido observadas diferenças entre os profissionais ou entre as cidades em que estes atendem nas demais questões. Conclusão: A amostra, equilibradamente, trabalha com um fonoaudiólogo de confiança e julga importante para adesão ao tratamento atuar no mesmo espaço físico. As indicações são realizadas logo após a avaliação odontológica, sendo a falta de tempo o maior motivo apresentado para não buscarem o tratamento, apesar de pacientes com condições financeiras favoráveis aderirem facilmente à fonoterapia. Os encaminhamentos são realizados geralmente pelas mesmas razões.
\end{abstract}

Descritores: Fonoterapia; Mercado de trabalho; Ortodontia; Odontopediatria; Questionários

\section{INTRODUÇÃO}

A interação entre a Fonoaudiologia e a Odontologia vem se aperfeiçoando satisfatoriamente, fundamentada, entre outros aspectos, no interesse partilhado na saúde e fisiologia do sistema estomatognático ${ }^{(1)}$. Fazem parte desse sistema as estruturas orais estáticas e dinâmicas que, equilibradas e controladas pelo sistema nervoso central, serão responsáveis

Trabalho realizado no CEFAC - Saúde e Educação - Belo Horizonte (MG), Brasil.

(1) Especialista em Motricidade Orofacial; Fonoaudióloga da Associação de Proteção ao Excepcional - APEX - Belo Horizonte (MG), Brasil.

(2) Especialista em Motricidade Orofacial; Fonoaudióloga da Prefeitura Municipal de Itabira - Itabira (MG), Brasil.

(3) Pós-graduanda em Distúrbios da Comunicação Humana pela Universidade Federal de São Paulo - UNIFESP - São Paulo (SP), Brasil; Professora Assistente do Departamento de Fonoaudiologia da Universidade Federal de Minas Gerais - UFMG - Belo Horizonte (MG), Brasil.

Endereço para correspondência: Cibele Pires de Moraes Varandas. R. Marechal Bittencourt, 368/101, Gutierrez, Belo Horizonte - MG, CEP 30430200. E-mail: cilemo_pos@yahoo.com.br

Recebido em: 10/9/2007; Aceito em: 1/7/2008 pelo funcionamento harmônico da face. Por ser um sistema interdependente e desenvolver funções comuns, qualquer alteração em um de seus componentes acarretará um desarranjo geral. Porém, não se justifica que os fonoaudiólogos conheçam apenas as estruturas ativas, assim como o dentista conheça apenas as estruturas passivas ${ }^{(2)}$. Nos dias de hoje, é de extrema importância a atuação interdisciplinar, que inclua profissionais da saúde com diferentes áreas de atuação e que estejam integrados no propósito de beneficiar o paciente.

A atuação ortodontista/fonoaudiólogo está intimamente relacionada, uma vez que a intervenção de um interfere e depende diretamente do trabalho do outro. Estes profissionais devem buscar um atendimento globalizado, eficiente e adequado, com intuito de proporcionar uma melhor qualidade de vida ao paciente ${ }^{(3)}$. Muitos dentistas se dizem convencidos de que não existe um resultado satisfatório em um tratamento ortodôntico, sem que haja um trabalho associado com a Fonoaudiologia, quando existe uma alteração miofuncional, sendo que o ideal seria que esse trabalho fosse realizado no mesmo espaço físi$\mathrm{Co}^{(4)}$. É importante também que se estabeleça um padrão entre os registros destes profissionais ${ }^{(5)}$. De acordo com ortodontistas 
e ortopedistas faciais, os fonoaudiólogos devem, sempre que estiverem tratando um paciente em conjunto, entender quais os objetivos do tratamento odontológico proposto ${ }^{(2)}$.

Nos tratamentos ortodônticos/ortopédicos nos quais é necessário o restabelecimento do equilíbrio da musculatura orofacial, o auxílio do fonoaudiólogo será fundamental, uma vez que forma e função estão intimamente correlacionadas. Entretanto, muitas vezes, é necessário aguardar a intervenção odontológica ou de outros profissionais envolvidos no processo.

Estudo aponta a postura de língua como uma das principais causas da ocorrência de recidivas orais ${ }^{(6)}$. Observa-se que se o paciente após o término do tratamento ortodôntico/ortopédico não corrigir a respiração oral, as chances de recidiva serão grandes. Desta forma, antes do início de qualquer intervenção, o paciente deve ser informado da necessidade de tratar a respiração oral provavelmente com médicos e fonoaudiólogos, para obter resultados estáveis ${ }^{(2)}$.

O fonoaudiólogo, portanto, por meio da terapia miofuncional, promove o tratamento das alterações musculares e funcionais orofaciais, favorecendo a estabilidade dos casos tratados por ortodontistas/ortopedistas funcionais dos maxilares e odontopediatras, buscando evitar recidivas oclusais, após a retirada do aparelho ${ }^{(6)}$. Portanto, conhecer a percepção que parceiros de trabalho têm em relação à nossa profissão poderá auxiliar a Fonoaudiologia a pensar em sua atuação no mercado de trabalho, especialmente comparando-se dados de uma capital e do interior. Torna-se fundamental, também, esclarecer qual das duas especialidades, ortodontistas e odontopediatras, possui maior conhecimento sobre o trabalho fonoaudiológico, para que a comunidade científica identifique o foco de um trabalho de conscientização, tornando possível uma maior aproximação entre profissionais e um trabalho efetivamente interdisciplinar.

Dessa forma, o objetivo deste trabalho foi verificar a percepção de ortodontistas e odontopediatras de Belo Horizonte e Itabira, sobre a adesão ao tratamento fonoaudiológico.

\section{MÉTODOS}

Este trabalho, caracterizado por um estudo transversal, foi realizado em consultórios particulares e clínicas de ortodontistas e odontopediatras das cidades de Belo Horizonte e Itabira, no Estado de Minas Gerais, tendo sido aprovado pelo Comitê de Ética em Pesquisa do CEFAC, sob o no 47/06.

O Conselho Regional de Odontologia foi contactado e obteve-se uma listagem dos profissionais cadastrados. Por meio desta listagem foram selecionados 30 ortodontistas e 30 odontopediatras. A escolha destes profissionais foi realizada, obedecendo-se à ordem da listagem até que atingisse 15 profissionais de cada área, em cada cidade, dispostos a colaborar com a pesquisa.

Como critérios de inclusão dos indivíduos no estudo, foram considerados apenas profissionais que tivessem concluído curso de especialização em Ortodontia ou Odontopediatria e que atuassem em Belo Horizonte e/ou Itabira. Foram excluídos os profissionais que não atuavam mais em sua área de especialização.

Os profissionais selecionados responderam a um questionário (Anexo 1) composto por 11 questões fechadas, com perguntas idênticas para ambas as especialidades, sobre como realizam os encaminhamentos para fonoterapia e como seus pacientes percebem os mesmos.

A partir dos resultados coletados foi realizado em estudo estatístico, empregando-se o teste Qui-quadrado com um nível de significância de 5\%, buscando-se comparar os resultados entre as categorias profissionais e entre as cidades pesquisadas.

\section{RESULTADOS}

Participaram deste estudo, 60 indivíduos, sendo 34 do gênero feminino e 26 do masculino, com média de idade de 35,8 anos. Os resultados foram analisados de acordo com as respostas dos 15 odontopediatras e 15 ortodontistas da cidade de Belo Horizonte e dos 15 odontopediatras e 15 ortodontistas da cidade de Itabira, ambas no Estado de Minas Gerais.

Da amostra estudada, $100 \%$ dos especialistas das duas cidades afirmaram considerar importante a atuação fonoaudiológica junto à sua especialidade e encaminhar pacientes para fonoterapia.

A freqüência com que os pacientes costumam precisar da intervenção fonoaudiológica pode ser observada na Tabela 1 e a frequiência em que os pacientes procuram o fonoaudiológo após encaminhamento encontra-se na Tabela 2.

Observou-se que, $80 \%$ dos odontopediatras e $66,7 \%$ dos ortodontistas da cidade de Belo Horizonte trabalham em parceria com um fonoaudiólogo de confiança. Em Itabira, 60\% dos odontopediatras e $80 \%$ dos ortodontistas possuem um profissional de referência para encaminharem os seus pacientes. Não se observou diferença significante entre as cidades $(p=0,774)$ ou entre os profissionais $(p=0,774)$.

A existência ou não de um fonoaudiólogo na clínica dos especialistas foi avaliada. Em Belo Horizonte, $80 \%$ dos odontopediatras e 73,3\% dos ortodontistas não possuem um fono-

Tabela 1. Freqüência com que os pacientes necessitam da intervenção fonoaudiológica

\begin{tabular}{|c|c|c|c|c|c|c|c|c|c|c|}
\hline \multirow[b]{3}{*}{ Freqüência } & \multicolumn{4}{|c|}{ Odontopediatra } & \multicolumn{4}{|c|}{ Ortodontista } & & \\
\hline & \multicolumn{2}{|c|}{$\mathrm{BH}$} & \multicolumn{2}{|c|}{ Itabira } & \multicolumn{2}{|c|}{$\mathrm{BH}$} & \multicolumn{2}{|c|}{ Itabira } & \multicolumn{2}{|c|}{ Valor-p* } \\
\hline & $\mathrm{n}$ & $\%$ & $\mathrm{n}$ & $\%$ & $n$ & $\%$ & $\mathrm{n}$ & $\%$ & Cidades & Profissionais \\
\hline Algumas vezes & 10 & $66,7 \%$ & 8 & $53,3 \%$ & 8 & $53,3 \%$ & 8 & $53,3 \%$ & & \\
\hline Freqüentemente & 5 & $33,3 \%$ & 5 & $33,3 \%$ & 6 & $40,0 \%$ & 7 & $46,7 \%$ & 0,550 & 0,389 \\
\hline Raramente & 0 & $0,0 \%$ & 2 & $13,3 \%$ & 1 & $6,7 \%$ & 0 & $0,0 \%$ & & \\
\hline
\end{tabular}

*Teste Qui-quadrado 
Tabela 2. Freqüência com que os pacientes procuram o fonoaudiólogo após encaminhamento

\begin{tabular}{|c|c|c|c|c|c|c|c|c|c|c|}
\hline \multirow[b]{3}{*}{ Freqüência } & \multicolumn{4}{|c|}{ Odontopediatra } & \multicolumn{4}{|c|}{ Ortodontista } & & \\
\hline & \multicolumn{2}{|c|}{$\mathrm{BH}$} & \multicolumn{2}{|c|}{ Itabira } & \multicolumn{2}{|c|}{$\mathrm{BH}$} & \multicolumn{2}{|c|}{ Itabira } & \multicolumn{2}{|c|}{ Valor-p* } \\
\hline & $\mathrm{n}$ & $\%$ & $\mathrm{n}$ & $\%$ & $\mathrm{n}$ & $\%$ & $\mathrm{n}$ & $\%$ & Cidades & Profissionais \\
\hline Algumas vezes & 7 & $46,7 \%$ & 8 & $53,3 \%$ & 6 & $40,0 \%$ & 7 & $46,7 \%$ & & \\
\hline Freqüentemente & 4 & $26,7 \%$ & 4 & $26,7 \%$ & 5 & $33,3 \%$ & 6 & $40,0 \%$ & 0,548 & 0,385 \\
\hline Raramente & 4 & $26,7 \%$ & 3 & $20,0 \%$ & 4 & $26,7 \%$ & 2 & $13,3 \%$ & & \\
\hline
\end{tabular}

* Teste Qui-quadrado

audiólogo trabalhando na mesma clínica. Em Itabira, 93,3\% dos odontopediatras e ortodontistas, também não possuem um profissional da Fonoaudiologia atuando no mesmo espaço físico. Novamente, não foram observadas diferenças entre as cidades $(p=0,071)$ ou entre profissionais $(p=0,071)$.

Quanto ao espaço físico, 80\% dos odontopediatras e 73,3\% dos ortodontistas da cidade de Belo Horizonte acreditam que o fato do fonoaudiólogo trabalhar no mesmo espaço físico aumentaria a adesão ao tratamento. Em Itabira, 100\% dos odontopediatras e $86,7 \%$ dos ortodontistas afirmaram acreditar que a proximidade física aumentaria a adesão, sendo o p-valor $=0,301$ entre profissionais e $\mathrm{p}=1,000$ entre as cidades.

$\mathrm{O}$ momento em que os especialistas das duas cidades encaminham seus pacientes para o tratamento fonoaudiológico, foi pesquisado na oitava pergunta. As respostas obtidas encontram-se na Tabela 3. As justificativas que os pacientes apresentam aos profissionais para não procurar o tratamento fonoaudiológico indicado encontram-se na Tabela 4.

Para 93,3\% dos odontopediatras e $80 \%$ dos ortodontistas de Belo Horizonte, pacientes com boas condições financeiras aderem ao tratamento fonoaudiológico. Dentre os profissio- nais de Itabira, 86,7\% dos odontopediatras e ortodontistas acreditam que a situação econômica boa é favorável à adesão ao tratamento. Mais uma vez não foram observadas diferenças entre as cidades $(p=0,718)$ e entre os profissionais $(p=0,718)$

Os motivos mais freqüentes dos encaminhamentos para a Fonoaudiologia podem observados na Figura 1.

\section{DISCUSSÃO}

A necessidade da integração profissional como a melhor forma de aperfeiçoar os processos de diagnóstico e intervenção é amplamente discutida na literatura ${ }^{(2,7-8)}$. Na prática clínica, essa demanda também pode ser observada. No presente estudo, todos os profissionais das duas cidades afirmaram considerar a atuação fonoaudiológica junto à sua especialidade importante, encaminhando seus pacientes para fonoaudiólogos.

A maior parte dos entrevistados relatou que seus pacientes necessitam de intervenção fonoaudiológica com freqüência moderada (algumas vezes). Esses dados concordam com a literatura, já que vários autores ${ }^{(4,6)}$ associam os distúrbios miofuncionais orofaciais e cervicais às recidivas, apesar de alguns

Tabela 3. Momento em que os profissionais geralmente realizam os encaminhamentos

\begin{tabular}{|c|c|c|c|c|c|c|c|c|c|c|}
\hline \multirow[b]{3}{*}{ Momento } & \multicolumn{4}{|c|}{ Odontopediatra } & \multicolumn{4}{|c|}{ Ortodontista } & & \\
\hline & \multicolumn{2}{|c|}{$\mathrm{BH}$} & \multicolumn{2}{|c|}{ Itabira } & \multicolumn{2}{|c|}{$\mathrm{BH}$} & \multicolumn{2}{|c|}{ Itabira } & \multicolumn{2}{|c|}{ Valor-p* } \\
\hline & $\mathrm{n}$ & $\%$ & $\mathrm{n}$ & $\%$ & $\mathrm{n}$ & $\%$ & $\mathrm{n}$ & $\%$ & Cidades & Profissionais \\
\hline Após a avaliação & 12 & $80,0 \%$ & 11 & $73,3 \%$ & 10 & $66,7 \%$ & 12 & $80,0 \%$ & & \\
\hline Durante o tratamento & 9 & $60,0 \%$ & 5 & $33,3 \%$ & 11 & $73,3 \%$ & 12 & $80,0 \%$ & & \\
\hline Após o término tratamento & 1 & $6,7 \%$ & 2 & $13,3 \%$ & 6 & $40,0 \%$ & 4 & $26,7 \%$ & 0,319 & 0,352 \\
\hline Após recidiva & 1 & $6,7 \%$ & 2 & $13,3 \%$ & 0 & $0,0 \%$ & 0 & $0,0 \%$ & & \\
\hline Outros & 1 & $6,7 \%$ & 0 & $0,0 \%$ & 0 & $0,0 \%$ & 0 & $0,0 \%$ & & \\
\hline
\end{tabular}

* Teste Qui-quadrado

Tabela 4. Justificativa dos pacientes para não procurar o tratamento fonoaudiológico

\begin{tabular}{|c|c|c|c|c|c|c|c|c|c|c|}
\hline \multirow[b]{3}{*}{ Justificativa } & \multicolumn{4}{|c|}{ Odontopediatra } & \multicolumn{6}{|c|}{ Ortodontista } \\
\hline & \multicolumn{2}{|c|}{$\mathrm{BH}$} & \multicolumn{2}{|c|}{ Itabira } & \multicolumn{2}{|l|}{$\mathrm{BH}$} & \multicolumn{2}{|c|}{ Itabira } & \multicolumn{2}{|l|}{ Valor-p* } \\
\hline & $\mathrm{n}$ & $\%$ & $\mathrm{n}$ & $\%$ & $\mathrm{n}$ & $\%$ & $\mathrm{n}$ & $\%$ & Cidades & Profissionais \\
\hline Falta de tempo & 11 & $73,3 \%$ & 12 & $80,0 \%$ & 12 & $80,0 \%$ & 15 & $100 \%$ & & \\
\hline Questão financeira & 4 & $26,7 \%$ & 4 & $26,7 \%$ & 4 & $26,7 \%$ & 2 & $13,3 \%$ & 0,652 & 0,652 \\
\hline Não julgam importante & 6 & $40,0 \%$ & 3 & $20,0 \%$ & 3 & $20,0 \%$ & 4 & $26,7 \%$ & & \\
\hline
\end{tabular}

* Teste Qui-quadrado 


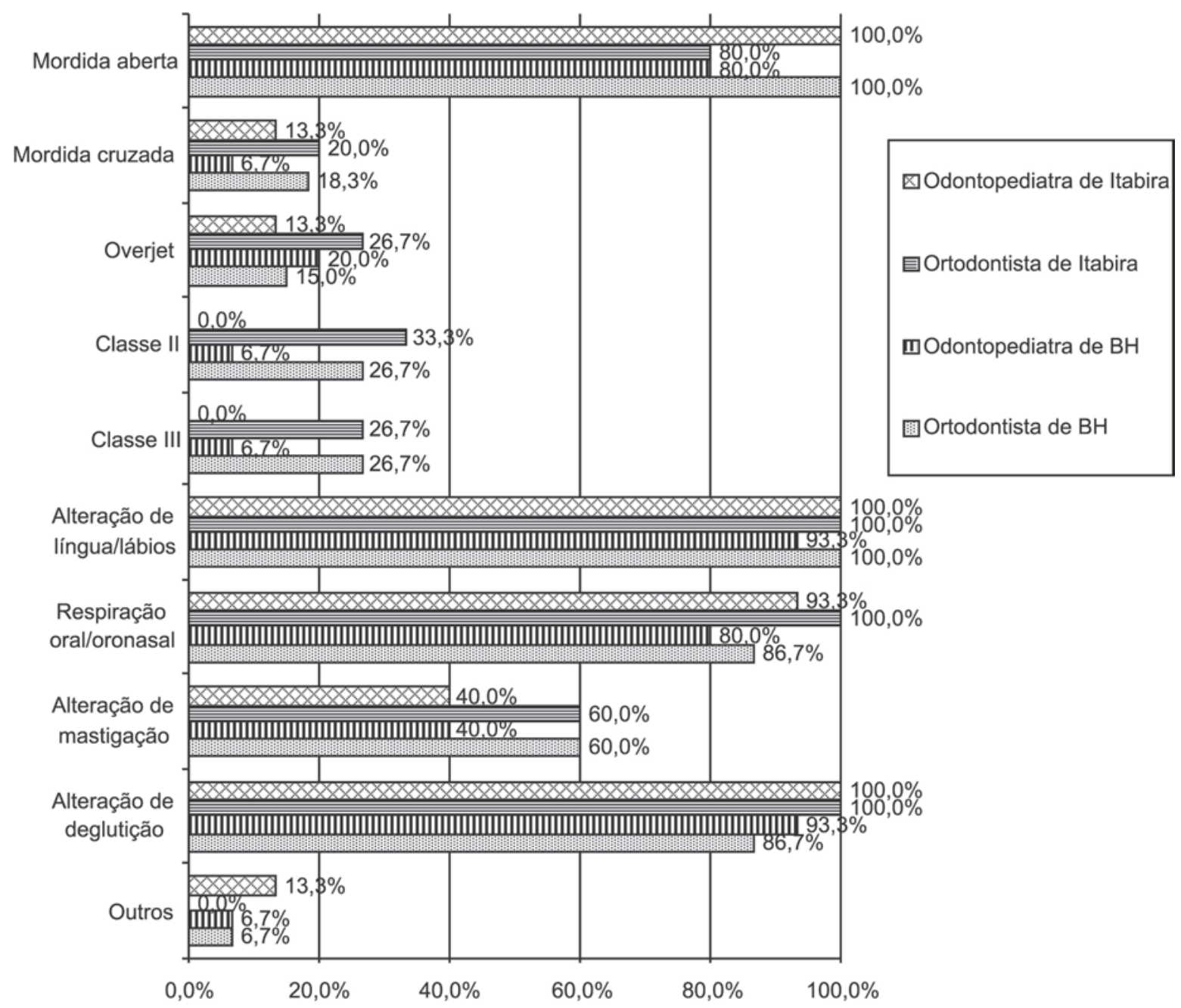

Figura 1. Razão para o encaminhamento

fonoaudiólogos acreditarem haver resistência dos dentistas nos encaminhamentos ${ }^{(9)}$. Observou-se que um número maior de ortodontistas indicou uma necessidade mais elevada de fonoterapia; entretanto, não foram observadas diferenças significantes entre profissionais ou entre as cidades pesquisadas. Estudos $^{(10)}$ indicam que a função dos aparelhos ortodônticos sobre o sistema estomatognático é limitada, alterando apenas a disposição dos arcos dentários, enquanto a terapia fonoaudiológica irá trabalhar a reabilitação muscular e funcional.

Em relação à freqüência com que seus pacientes buscam o tratamento fonoaudiológico indicado, a maioria dos pesquisados relatou, de forma equilibrada entre as cidades e categoria profissional, uma busca mediana (algumas vezes). Estudos indicam existir certa resistência dos pacientes em realizar uma avaliação fonoaudiológica solicitada por ortodontistas e odontopediatras $^{(9)}$.

A maioria dos profissionais indicou conhecer um fonoaudiólogo de referência para indicação dos casos. Conhecer o profissional facilita a comunicação, beneficiando as duas áreas e especialmente o paciente. Esse contato maior, mesmo que não ocorra no mesmo espaço físico, pode auxiliar na padronização dos registros ${ }^{(5)}$ e no entendimento dos objetivos terapêuticos ${ }^{(2)}$. De acordo com estudo ${ }^{(4)}$, a proximidade física seria um importante fator para os ortodontistas. Na presente pesquisa, a grande maioria dos entrevistados relatou não trabalhar no mesmo espaço que um fonoaudiólogo, apesar de acreditarem que esse fato aumentaria a adesão ao tratamento fonoaudiológico, independente de residirem na capital ou em uma cidade do interior. Esta informação poderá auxiliar profissionais que estejam iniciando sua prática clínica na escolha do local de trabalho, apesar de ser necessária, ainda, uma confirmação da hipótese.

Grande parte dos pesquisados das duas cidades informaram encaminhar seus pacientes para fonoaudiólogos, logo após a avaliação. A decisão sobre o momento de iniciar uma intervenção fonoaudiológica, ou mesmo se esta é necessária, deve ser tomada em conjunto, entre os profissionais ${ }^{(11)}$. Apesar de se considerar que cada paciente apresente uma necessidade diferenciada $^{(12)}$, o encaminhamento no início do tratamento odontológico permite uma discussão melhor dos casos, evitando-se intervenções tardias. É consenso que a correção ortodôntica só poderá manter-se adequada se estiver em harmonia com o equilíbrio da musculatura do paciente. Desta forma, deve-se aguardar a alta fonoaudiológica para a alta ortodôntica final, já que a mesma necessita de restabelecimento do equilíbrio muscular ${ }^{(8)}$. Cabe ressaltar que os odontopediatras de Itabira 
realizam poucos encaminhamentos ao longo do tratamento odontológico e que mais ortodontistas realizam encaminhamento após o término do tratamento. Mais uma vez não se observou qualquer associação estatística.

A maior causa levantada no estudo para a não adesão ao tratamento fonoaudiológico foi falta de tempo. Apesar do real excesso de atividades das quais as crianças nos dias atuais participam, e do fato de muitos pais não terem disponibilidade, em decorrência da jornada de trabalho, a gravidade da doença, o gênero e a restrição econômica são os fatores considerados na literatura para adesão/não adesão ao tratamento fonoaudiológico ${ }^{(13)}$. Entretanto, os pacientes podem omitir a verdadeira razão para não procurarem o tratamento indicado, uma vez que os próprios pesquisados relataram que aqueles com boas condições financeiras geralmente aderem à fonoterapia. Acredita-se que, muitas vezes, a não compreensão da importância da Fonoaudiologia, associada à questão financeira, pode influenciar nesta decisão, apesar do presente estudo ter sido conduzido em consultórios particulares. Muitas famílias alegam já realizar um gasto extra com o tratamento ortodôntico que, contrariamente à intervenção odontopediátrica, costuma ser mais prolongado. Apesar de não terem sido observadas diferenças significantes, odontopediatras de BH relataram que: "não julgam importante".

Ao se analisar os dados foi verificado que existe uma semelhança muito grande entre os motivos de encaminhamento tanto em relação às especialidades quanto às cidades, apesar dos casos de más oclusões de Angle serem pouco encaminhados, especialmente por odontopediatras. Apesar de se poder supor que os odontopediatras encaminhem, inicialmente, os casos de alteração oclusal para os ortodontistas, este fato não foi verificado em relação às mordidas abertas.

Portanto, os distúrbios miofuncionais orofaciais, de uma forma geral, se constituíram como um motivo bem estabelecido de indicação para tratamento fonoaudiológico na amostra estudada. Entretanto, em relação às más oclusões, observou-se grande discrepância entre as mordidas abertas e as demais más oclusões. Cabe ressaltar que, em muitos casos, se o paciente não apresentar a forma adequada, é praticamente impossível ou limitada uma atuação fonoaudiológica. Todavia, na prática clínica, observa-se que freqüentemente pacientes com mordida aberta anterior são encaminhados, antes da correção ortodôn- tica para fonoterapia ${ }^{(2)}$.

Assim, verifica-se a necessidade de ampla divulgação da abrangência da atuação fonoaudiológica na área de Motricidade Orofacial, incluindo as possíveis limitações. O papel da Fonoaudiologia não se encontra totalmente definido para ortodontistas e odontopediatras ${ }^{(14-15)}$, sendo observadas resistências no encaminhamento ${ }^{(9)}$ ou mesmo dificuldades no que se refere a que casos necessitam de intervenção fonoaudiológica e o momento adequado para que a mesma ocorra ${ }^{(16)}$. Portanto, cabe aos fonoaudiólogos que atuam em Motricidade Orofacial buscar reverter este quadro, esclarecendo os dentistas acerca deste campo de atuação.

Por meio deste estudo pôde-se observar que não ocorreram diferenças significantes entre as opiniões de ortodontistas e odontopediatras ou entre as cidades investigadas, apesar das realidades bastante distintas. Cabe ressaltar que a amostra apresentada foi reduzida, limitando a análise estatística. Outras pesquisas deverão ser conduzidas para que os dados possam ser confirmados ou refutados. Outra limitação do presente trabalho refere-se à ausência de estudos sobre adesão à fonoterapia, impedindo, assim, a comparação dos achados.

\section{CONCLUSÃO}

$\mathrm{Na}$ amostra investigada, tanto ortodontistas quanto odontopediatras das cidades de Belo Horizonte e Itabira, em Minas Gerais, consideram importante a atuação fonoaudiológica, encaminhando casos com alguma frequiência para terapia. Entretanto, apenas uma parte dos pacientes a buscam. Geralmente, os profissionais trabalham com um fonoaudiólogo de confiança, apesar de não atuarem no mesmo local, mesmo julgando este fato importante para a adesão ao tratamento fonoaudiológico. As indicações são realizadas logo após a avaliação odontológica, sendo a falta de tempo o principal motivo alegado para não buscarem o fonoaudiólogo, apesar de pacientes com condições favoráveis aderirem facilmente ao tratamento. Os encaminhamentos são realizados geralmente pelo mesmo motivo, exceto nos casos de más oclusões de Angle, que não são encaminhadas por odontopediatras de Itabira.

Não foram observadas diferenças entre as respostas de odontopediatras e ortodontistas ou entre as cidades em que estes profissionais atuam. 


\begin{abstract}
Purpose: To verify the perception of orthodontists and pediatric dentists from Belo Horizonte and Itabira concerning adhesion to speech therapy. Methods: A transversal study was carried out by using a questionnaire with 30 orthodontists and 30 pediatric dentists, half from each city. Statistical analysis used the Chi-square test. Results: The entire group of professional considered speech therapy important and reported to frequently refer their patients to this practice. From the sample, 58.3\% of the professionals reported that their patients sometimes need speech therapy; $46.7 \%$ informed that sometimes patients also look for a speech therapist, and $70.0 \%$ have a trustworthy speech therapist to whom they usually refer patients to. It was verified that $85 \%$ of the sample informed that they do not work with speech therapists in the same work environment, and $85 \%$ believe that sharing the same space would increase the adhesion to therapy. To 75\%, the patients' referral is usually carried out after the evaluation, and $83.3 \%$ informed that patients do not look for treatment due to lack of time, although $86.7 \%$ affirmed that patients with good financial condition usually do adhere to speech therapy. The referrals are usually made for the same reason, except in cases regarding malocclusions. In the other questions, no differences were found between pediatric dentists and orthodontists or between cities. Conclusion: The professionals interviewed deal with a trustworthy speech therapist and judge that sharing the same work environment is important to increase patients' adhesion to treatment. The patients' referrals are usually made right after the odontological evaluation, and lack of time is the main reason why they do not look for a speech therapist, although patients with good financial situation easily adhere to treatment. Referrals are usually made because of the same reasons.
\end{abstract}

Keywords: Speech therapy; Job market; Orthodontics; Pediatric dentistry; Questionnaires

\section{REFERÊNCIAS}

1. Campos LM, Munhoz SRM, Imparato JCP, Echeverrias SRPS, Servilha EAM. A interação entre odontologia e fonoaudiologia na anodontia parcial verdadeira: relato de caso. J Bras Fonoaudiol. 2002;3(11):97102.

2. Marchesan IQ, Sanseverino NT. Conhecimentos essenciais para entender bem a relação entre fonoaudiologia e ortodontia/ortopedia facial: esclarecendo dúvidas sobre o trabalho conjunto. São José dos Campos: Pulso Editorial; 2004.

3. Melgaço CA, Di Ninno CQMS, Penna LM, Vale MPP. Aspectos ortodônticos/ortopédicos e fonoaudiológicos relacionados a pacientes portadores de fissuras labiopalatinas. J Bras Ortodon Ortop Facial. 2002;7(37):23-32.

4. Cavassani VGS, Ribeiro SG, Nemr NK, Greco AM, Köhle J, Lehn CN. Hábitos orais de sucção: estudo piloto em população de baixa renda. Rev Bras Otorrinolaringol. 2003;69(1):106-10.

5. Korbmacher H, Kahl-Nieke B. Optimizing interdisciplinary cooperation for patients with orofacial dysfunctions. Presentation of an interdisciplinary diagnostic referral sheet. J Orofac Orthop. 2001;62(3):246-50.

6. Comin I, Passos Filho LP. Ortopedia funcional dos maxilares e fonoaudiologia: uma possibilidade terapêutica multidisciplinar. Rev Dent Press Ortodon Ortop Maxilar. 1999;4(4):63-70.

7. Jorge TM, Duque C, Berretin-Felix G, Costa B, Gomide MR. Hábitos bucais - interação entre odontopediatra e fonoaudiologia. JBP J Bras Odontopediatr Odontol Bebe. 2002;5(26):342-50.

8. Araújo RJH, Araújo RJA, Camargo MEPS, Periotto MC. Integração ortodontia-ortopedia funcional dos maxilares e fonoaudiologia. Rev Paul Odontol. 2000;22(4):24-9.
9. Amaral EC, Bacha SMC, Ghersel ELA, Rodrigues PMI. Inter-relação entre a odontologia e a fonoaudiologia na motricidade orofacial. Rev CEFAC. 2006;8(3):337-51.

10. Maciel CTV, Leite ICG. Aspectos etiológicos da mordida aberta anterior e suas implicações nas funções orofaciais. Pro-Fono. 2005;17(3):293302.

11. Bacha SMC. Deglutição: automatização é possível? In: Comitê de Motricidade Orofacial da Sociedade Brasileira de Fonoaudiologia. Motricidade orofacial: como atuam os especialistas. São José dos Campos: Pulso Editorial; 2004. p. 77-86.

12. Medeiros AMC, Medeiros M. Motricidade orofacial: inter-relação entre fonoaudiologia e odontologia. São Paulo: Lovise; c2006.

13. Chatkin JM, Cavalet-Blanco D, Scaglia NC, Tonietto RG, Wagner MB, Fritscher CC. Adesão ao tratamento de manutenção em asma (estudo ADERE). J Bras Pneumol. 2006;32(4):277-83.

14. Mendes ACS, Costa AA, Nemr K. O papel da fonoaudiologia na ortodontia e odontopediatria: avaliação do conhecimento dos odontólogos especialistas. Rev CEFAC. 2005;7(1):60-7.

15. Rodrigues HOSN, Faria SR, Paula FSG, Motta AR. Ocorrência de respiração oral e alterações miofuncionais orofaciais em sujeitos em tratamento ortodôntico. Rev CEFAC. 2005;7(3):356-62.

16. Coutinho PMC, Amaral KM, Lemes SS, Davila VL, Vieira MEMG. Interfaces entre as atuações terapêuticas fonoaudiológicas e odontológicas. Rev CEFAC. 2003;5(2):131-7. 
Anexo 1. Questionário aplicado aos ortodontistas e odontopediatras

\section{Questionário}

Sexo: ( ) Masculino ( ) Feminino

Idade:

1. Você considera importante a atuação fonoaudiológica junto à sua especialidade? () Sim () Não

2. Você encaminha seus pacientes para a Fonoaudiologia? () Sim ( ) Não

3. Caso encaminhe, com qual freqüência os pacientes costumam precisar da intervenção fonoaudiológica?
( ) Raramente
( ) Algumas vezes
( ) Frequentemente

4. Após seu encaminhamento com qual freqüência seus pacientes costumam procurar o fonoaudiólogo?
() Raramente
( ) Algumas vezes
( ) Frequentemente

5. Você possui algum fonoaudiólogo de sua confiança para encaminhar seus pacientes? ( ) Sim ( ) Não

6. Existem fonoaudiólogos em sua clínica/consultório? ( ) Sim ( ) Não

7. Você acredita que o fato do fonoaudiólogo trabalhar no mesmo espaço físico aumenta a adesão do paciente ao tratamento?

( ) $\operatorname{Sim}$ ( ) Não

8. Em qual momento você costuma encaminhar seus pacientes para o tratamento fonoaudiológico? (caso julgue necessário marque mais de uma opção).
( ) Após a avaliação
( ) Durante o tratamento
( ) Após o término de seu tratamento

( ) Após recidiva ( ) Não encaminha ( ) Outros:

9. Qual(is) justificativa(s) seus pacientes apresentam para não procurar o tratamento fonoaudiológico?
( ) Falta de tempo
( ) Questão financeira
( ) Não julgarem importante.

10. Os pacientes com condições financeiras favoráveis, em sua maioria, aderem ao tratamento fonoaudiológico?

( ) $\operatorname{Sim}($ ) Não

11. Assinale as situações em que seus pacientes são encaminhados à Fonoaudiologia: (caso julgue necessário marque mais de uma opção)
( ) Mordida aberta
( ) Mordida cruzada
( ) Overjet
( ) Classe II
( ) Classe III
( ) Alteração de postura de língua/lábio
( ) Respiração oral/oronasal ( ) Alteração de mastigação

( ) Alteração de deglutição

( ) Outros: 